\title{
Particularidades del Acceso al Arbitraje Internacional en los Tratados Bilaterales de Inversión suscritos por la República del Ecuador
}

C. Ignacio Suarez Anzorena

\begin{abstract}
SUMARIO
Introducción. 1. Anatomía de un TBI. 2. Solución de Disputas Inversor-Estado. 2.1. Consideraciones iniciales. 2.2.- Periodos de espera/negociación. 2.3. Opciones jurisdiccionales. 2.4. Jurisdicciones disponibles. 2.5. Extensión material de la cláusula de solución de controversias. 3. Operación de la cláusula de Nación más favorecida en materia procesal. 4. Consideraciones finales.
\end{abstract}

\section{Introducción}

\section{La relevancia de los TBIs en el Derecho Internacional Económico Contemporáneo}

El presente trabajo tiene como objetivo brindar una introducción a los aspectos operativos de los mecanismos de solución de controversias entre inversores y estados que establecen los Tratados Bilaterales de Inversión ("TBIs"). En particular, la atención se centrará en el rol que el arbitraje internacional cumple en tal contexto y en los requisitos que condicionan el acceso a tan especial jurisdicción. 
Los TBIs se han transformado en uno de los instrumentos centrales del Derecho Internacional económico contemporáneo desde que, la por entonces República Federal de Alemania tomó la iniciativa de promover este tipo de acuerdos interestatales allá por 1959'. Todo comenzó -o al menos tomó una forma similar a la actual- en 1957, cuando un grupo de empresarios alemanes, reunidos en Colonia, publicaron un proyecto dirigido a fomentar la protección de sus inversiones en el extranjero, el cual se denominó International Convention for the Mutual Protection of Property Rights in Foreign Countries².

Mucha agua ha corrido bajo el puente desde ese entonces y los TBIs se han transformado en la herramienta jurídica internacional más difundida para promover los flujos internacionales de capital. La fórmula sobre la que se asienta tal vocación de promoción es muy simple: brindar a los inversores ciertas garantías de trato sustancial y procesal a los fines de facilitar la administración del riesgo político asociado con los proyectos de inversión en jurisdicciones extranjeras. Tal riesgo político tiene componentes variados, siendo los más importantes: i) la incertidumbre regulatoria propia de incursionar en sistemas legales desconocidos; y ii) los posibles déficits institucionales -o la percepción de éstos- en una cierta jurisdicción, lo cual incide en el riesgo de que el estado huésped interfiera con el uso y goce de una inversión sin brindar remedios adecuados.

En la medida que los TBIs sirven para administrar el riesgo político, el efecto esperado es que aumente el volumen de inversión por devenir al volverse viables ciertos proyectos que no lo eran previamente, y/o se reduzca el costo del capital relativo a los proyectos de inversión en la medida que se le da mayor certidumbre legal a su continuidad. Es importante entender, a estos fines, que un proceso de inversión está generalmente condicionado por una asimetría de información originaria: el inversor

1. Ver "The Proposed Convention to Protect Private Forcign Imesiment", Journal of Public Law, volume 9,1960 , p. 115

2. Ibid. p. 116. 
tiene capacidad de negociar hasta que invierte, pero luego de que instala riqueza en el estado huésped, queda a merced de sus instituciones y circunstancias, no sabiendo si en el mediano o largo plazo los compromisos asumidos y las expectativas creadas serán efectivamente honrados.

La siguiente información estadística ilustra acerca de la importancia de los TBIs en el Derecho Internacional económico contemporáneo3:

- Para fines del año 2008 se habían firmado un total de aproximadamente 2.676 TBIs.

- 483 TBIs , un $18 \%$ del total, tienen como parte a países de América Latina y el Caribe. Durante el año 2008, ésta fue la región menos activa en cuanto a la conclusión de nuevos TBIs.

- Los Tratados sobre Doble Imposición Tributaria, que constituyen el otro instrumento tradicional dirigido a facilitar los flujos de capitales internacionales, sumaban aproximadamente 2.805 para fines de 2008 .

- A fines de 2009 ya se habían verificado un total de 357 disputas entre inversores y estados con base en un TBI, de los cuales un $57 \%$ fueron iniciados desde a partir del año 2005. Del total de casos citados, 225 fueron registrados ante el Centro Internacional de Arreglo de Diferencias Relativas a Inversiones ("CIADI") 40 ante el

3. Ver publicación de la organización United Nations Conference on Trade And Development ("UNC. TAD"): "Recent Developments in International investment Agreements", (2008-June 2009), IIA Monitor 3 (2009).

4. CIADI es una organización intemacional que administra procedimientos de arbitraje y conciliación relativos a disputas entre partes estatales $\mathrm{e}$ inversores extranjeros. EI CIADI tiene origen en un tratado, cl Convenio sobre Arreglo de Diferencias Relativas a Inversiones entre Estados y Nacionales de Otros Estados ("Convenio CLADI"), el cual regula su funcionamiento, asi como también las obligaciones de los estados contratantes con relación al CIADI y a los procedimientos que éste administra. El Convenio CIADI fue concebido y redactado bajo los auspicios del Banco Internacional de Reconstrucción y Fomento (el "Banco Mundial") y sometido a consideración de los gobiernos miembros de tal organismo el $18 \mathrm{de}$ marzo de 1965. Entró en vigencia el 14 de octubre de 1966, cuando fue ratificado por 20 países. En el presenie, está en vigor con relación a más de 144 estados (para una lista de los miembros, véase: www.worldbank.org/icsid). El Convenio ClADI es uno de los instrumentos juridicos de Derecho Intemacional más exitosos del mundo contemp̧oráneo. La República del Ecuador se suscribió al Convenio CIADI el 15 de enero de 1986 y en febrero de ese mismo año entró 
Particularidades del acceso al Arbitraje Internacional en los Tratados Bilaterales

de Imersión suscritos por la Repuiblica del Ecuador

Mecanismo Complementario del mismo centro; 91 ante la Comisión de Naciones Unidas para el Derecho Mercantil Internacional ("CNUDMI" o "UNCITRAL", según sus siglas en inglés); 19 ante la Cámara de Comercio de Estocolmo ("CCE"); 8 casos fueron administrados por la Corte Permanente de Arbitraje de La Haya ("CPA"); 5 con la Cámara de Comercio Internacional ("CCl") y 1 en el Centro Regional de Arbitraje Comercial Internacional de El Cairo. Existen 4 casos más, de los cuales no se cuenta con información acerca de la reglamentación aplicable. ${ }^{5}$

La República del Ecuador ha sido uno de los protagonistas principales en este campo del derecho en los últimos años, habiendo suscrito mas de 20 TBIs, ${ }^{6}$ y habiendo estado involucrado en al menos 12 disputas. ${ }^{7}$ El gobierno de Ecuador fue condenado en los casos Occidental", Chevron', Repsol10 y Duke-

en vigencia. La Repuiblica del Ecuador ha denunciado cí Convenio CLADI en julio de 2009, denuncia ya se ha hecho efectiva a partir del 7 de enero de 2010.

5. Ver publicación de UNCTAD; "Latest Developments in Imestor-State Dispute Settement", (IIA Issues Note No I (2010) IIA.

6. Ver Tratados Bilaterales de Inversiön suscritos por la República del Ecuador en la base de datos de UNCTAD (http://www.unctadxi.org/templates/DocSearch 779.aspx.) y el Sistema de Información de Comercio Exterior ("SICE") de la Organizaciỏn de los Estados Americanos (htip://www.sice.oas.org/ctyindex/ECU/ECUBITs s. asp). Alli se hace referencia a los tratados firmodos con la República Argentina, firmado el 18/02/1994, en vigencia desde el 01/12/1995; con la República Alemana, firmudo el 21/03/1996, en vigencia desde el 12/02/1999; con la República de Bolivia, firmado el 25/05/1995, en vigencia desde el 15/08/1997; con el Gobiemo de Canada, firmado el 29/04/1996, en vigencia desde el 06/06/1997; con la República de Chile, firmado el 23/10/1993. en vigencia desde el 21/02/1996; con la República Popular China, firmado el 2I/03/1994, en vigencia desde 01/07/1997, con la República de Costa Rica, firmado el 06/12/2001 (no se cuenta con informaciön de que haya entrado en vigencia); con la República de El Salvador, firmado el 16/05/1994, en vigencia desde el 14/0L/1996, con el Reino de España, firmado el 26/06/1996, en vigencia desde el 18/06/1997; con las Estados Unidos de América, firmado el 27/08/1993, en vigencia desde el 11/05/1997; con la República Francesa, firmado cl 07/09/1994, en vigencia desde cl 10/06/1996; con la República de Nicaragua, firmado el 02/06/2000, en vigencia desde el 08/09/2002, con la República del Paraguay, firmado el 02/06/2000, en vigencia desde el 08/09/2002; con la República del Peri, firmado el 07/04/1999, en vigencia desde el 09/12/1999, con el Reino de los Paises Bajos, firmado el 27/06/1999, en vigencia desde el 01/07/2001; con el Gobierno de Rumania, firmado el 21/03/1996 (no se cuenta con información de que haya entraco en vigencia); con el Reino de Suecia, firmado el 01/05/2001, en vigencia desde el 31/05/2001; con la Confederación Suiza, firmado el 02/05/1968, en vigencia desde el 09/11/1969; con el Reino Unido de Gran Bretana e Irlanda del Norte, firmado el 10/05/1994, en vigencia desue 24/08/1995; con el Gobiemo de la República Dorninicana, firmado el 26/06/1998 (no se cuenta con información de que haya entrado en vigencia); con la República de Venezuela, firmado el 18/11/1993, en vigencia desde 01/02/1995. 
Ignacio Suarez

Electroquil" y obtuvo laudos favorables en los casos $\mathrm{MCI}{ }^{12}$, Encana ${ }^{13}$ y Empresa Eléctrica del Ecuador ${ }^{14}$. En el presente hay varios casos pendientes contra Ecuador. ${ }^{15}$

La República de Ecuador ha tomado medidas en los últimos tiempos para intentar reducir su exposición a este tipo de reclamos. En 2008 Ecuador denunció 9 TBIs, en su mayoría con pai-

7. Por cjemplo: Burlington Resouress Inc. y otros versis Repuiblica del Ecuador y Empresa Estatal Petrileos del Ecuador (PetroEcuador), [Caso CIADI No. ARB/08/5]; IBAf Horld Trade Corp. versus Repuiblica del Ecuador. [Caso CIADI No. ARB/02/10]; Noble Energy Inc. and Machala Power Cia. Lid. versus Repiblica del Ecuador y Conscjo Nacional de Electricidad, [Caso CIAD] No. ARB/0S/12].

8. En Occidental Exploration and Production Company versus Repiblica del Ectador, [Caso Corte de Arbitraje Internacional de Londres No. UN3467]. Ecuador fue condenado a pagar la suma de USS 75.074 .929 mas intereses y gastos legales. En Occidental Petroleum Corporation and Occidental Exploration and Prodiction Company versus Reprihlica del Ecuador, [Caso CLADI No. ARB/06/11], al momento de la preparación de éste articulo, no se ha dietado taudo final.

9. En Chevmn Corporation (USA) and Texaco Pctroleum Company (USA) versus Repuiblica del Ecuador; [Caso CNUDM1, laudo parcial de méritos de 30 de marzo de 2(1)10], el Tribunal decidió que Ecuador violó el Articulo II (7) del TBI y como consecuencia es resp̧onsable por los daños crussados a las demandantes.

10. En Repsol YPF Ecuador S.A. y atros versus Repuiblicu del Ecuador y Empresa Estatal Petróleos del Ecuador (Petroccttador), [Caso ClADI No ARB/01/10], el Tribunal condenó a Ecuador a pagar la suma de USS 13.684.279. La solicitud de anulación presentada por Ecuador en ese caso fue denegada.

11. En Duke Energy Electroquil Parmers df Electroquil S.d. versus República del Ecuador; [Caso CIADI No. ARB/04/19], Ecuador fue condenado a pagar la suma de USS 5.675.546 más intereses y costas legales.

12. En A.C.I. Power Group L.C. and New Turhine Inc. versus Repuiblica del Ecuador, [Caso CIADI No. ARB/(03/6], el Tribunal se acogió a la excepción internuesta por Ecuador sobre la competencia del Tribunal relativa a la irretroactividad del TB1.

13. En EnCana Corporation versus Repuiblica del Ecuador. [Caso CNUDMI, Coric de Arbitraje Internacional de Londres No. UN3481], el Tribunal declaró por unanimidad que los reclamos de la demandada estaban fucra de la esfera de su jurisdicción, excepto por el reclamo basado en el Articulo VIII del TBI, el cual rechazó de plano.

14. En Empresa Eléctrica del Ecuador, Inc. verstus Repuiblica del Ecuador, [Caso CIADI No. ARB/05/9]; el Tribunal determinó que no tenia competencia para entender en el caso.

15. Al momento de la preparación del presente trabajo, se encuentran pendientes ante el CIADI los casos: Occidental Petrolcum Corporation y Occidental Exploration and Production Company versus Republica de Ecuador: Afurphy Exploration and Production Company Internacional versus Republica del Ecuador; Burlington Resources Inc. versus Repuiblica del Ecuador (cl Tribunal, no obstante declarar su jurisdicción sobre el reclamo de expropiación, declaró falta de jurisdicción con respecto a los reclamos de las demandantes sobre falta de trato justo y equitativo, por trato arbitrario y por falta de protección y seguridad plenas bajo el TB1. Además, el Tribunal, declaró inadmisibles los reclamos de las demandantes por falta de protección y seguridad plenas bajo los Artículos 23 y 24 del TBI); Perenco Ecuador Limited versus Republica del Ecuador; Repsol YPF Ecuador S.A. y otros versus Reppiblica del Ecuador: Corporacioin Quiport S.A. y otros versus Repuiblica del Eciudor. 
ses de Latinoamérica. ${ }^{16}$ El 6 de julio de 2009 Ecuador denunció el Convenio sobre Arreglo de Diferencias Relativas a Inversiones entre Estados y Nacionales de Otros Estados ("Convenio CIADI"), luego de haber sido estado miembro por casi 24 años. ${ }^{17}$ Recientemente, la Corte Constitucional del Ecuador se pronunció acerca de la constitucionalidad de ciertas disposiciones de algunos TBIs en vigor a la luz del nuevo texto constitucional, concluyendo que sus cláusulas de solución de controversias no se ajustaban a la normativa doméstica. ${ }^{18}$

\section{Anatomía de un TBI}

-A los fines de apreciar debidamente la relevancia de la jurisdicción arbitral que consagran los TBIs en beneficio de los inversores, es importante contar con una visión, aunque sea básica y esquemática, de la estructura y de las principales características de tales instrumentos. Ello permite comprender los presupuestos que le sirven de antecedente a la disponibilidad de esta jurisdicción especial y, al mismo tiempo, dimensionar su rol como garantía procesal.

Una de las principales reglas a tener en cuenta al interpretarse los TBIs es la siguiente: parecen todos iguales, pero no lo son, y puede haber diferencias más o menos sutiles entre ellos con decisivas consecuencias frente a un caso determinado. Hecha esta advertencia, puede decirse, sin embargo, que los TBIs y los capítulos relativos a inversiones en los Tratados de

16. Los TBIs denunciados fueron los suscritos con: Cuba, El Salvador, Guatemala, Honduras, Nicaragua, República Dominicana, Paraguay, Uruguay y Rumania. Cabe notar que muchos TBI conticnen cláusulas de "ultra-actividad" que mantienen la vigencia de sus protecciones por un lapso determinado para la inversiones realizadas antes de la denuncia.

17. De conformidad con la Convención ClADI, una denuncia tiene cfectos luego de transcurridos 6 meses (art. 71 del Convenio ClADl). Previamente, el 4 de diciembre de 2007. Ecuador habia realizado una notificación al CIADI de conformidad con el articulo $25(4)$ de] Convenio CIADI el cual establece la posibilidad de que un estado parte notifique qué clase de diferencias aceptaria someter a la jurisdieciòn del Centro. No se considera.

18. La Corte Constitucional se pronunció con fecha 3 de agosto del 2010 acerca de los TBI con Alcmania y con el Reino Unido, $y$ con fecha 17 de agosto de 2010 acerea del TBIs con China y Finlandia. 
Libre Comercio ("TLCs") comparten una cierta anatomía estructural y presentan características comunes susceptibles de ser generalizadas, a los fines, al menos, de un análisis introductorio como el presente.

Un TBI puede dividirse, conceptualmente, en cuatro partes fundamentales:

i) Campo de aplicación: Cada TBI define qué tipo de derechos/activos califican como una inversión (campo de aplicación rationae materiae), qué tipo de personas físicas o jurídicas pueden invocar los derechos sustanciales y procesales que allí se consagran (campo de aplicación rationae personae) y cuál es el ámbito de aplicación temporal del tratado en lo que concierne a su vigencia y a las circunstancias temporales de la disputa (campo de aplicación rationae temporis).

ii) Normas relativas a la admisión de inversiones: Los TBIs, en general, establecen en que condiciones o circunstancias, o que tipo de inversiones son admisibles bajo sus términos. La mayoría de los TBIs sólo hacen referencia a la admisión de inversiones de conformidad con sus leyes y reglamentaciones ${ }^{19}$.

iii) Normas relativas al tratamiento de las inversiones: Este es el corazón sustancial de los TBIs. Implica la asunción de obligaciones específicas de trato con relación a las inversiones de inversores que cumplan con las reglas de admisión y califiquen dentro del campo de aplicación del TBI. La mayoría de los TBIs contienen obligaciones en materia de trato justo y equitativo, tratamiento nacional, tratamiento de nación más favorecida, prohibición de tratamiento arbitrario o discriminatorio que perjudique el uso y goce de la inversión, plena protección y seguridad, prohibición de expropiación, directa o indirecta, sin que medie debida compensación, tratamiento en supuestos de emergencias,

19. Ver, por ejemplo, TBI Ecuador - Argentina, articulo 2: "Cada Parte Contratante promoverá en su ferritorio las imversiones de imversores de la otra Parte Contratante y admitirá dichas inversiones conforme a sus leyes y reglamentaciones". 
catástrofes y guerras, y derechos en materia de repatriación de capital y utilidades. Algunos TBIs también obligan al estado huésped a observar las obligaciones que se hayan asumido con relación a inversiones (cláusulas paraguas). En el caso de los capítulos de inversiones, las obligaciones de trato sustancial pueden ser notoriamente más amplias e incluir cuestiones relativas a requisitos de desempeño para gozar de ciertas ventajas y prerrogativas, cuestiones relativas a la nacionalidad de los miembros de los órganos de administración de las sociedades, entre otras cuestiones.

iv) Normas sobre solución de controversins: Los TBIs contienen dos tipos de mecanismos de solución de controversias. El primero, para aquellas disputas que surgen entre los estados signatarios del TBI con motivo de la aplicación e interpretación de éste $^{20}$. El segundo, dirigido a resolver las disputas que puedan surgir entre quienes están habilitados para invocar la protección del TBI y el estado huésped de la inversión. Esta contribución está dirigida a analizar los aspectos operativos de este último mecanismo.

\section{SOlución de Disputas InVERsor-Estado}

\subsection{Consideraciones iniciales}

El remedio clásico que el Derecho Internacional consuetudinario provee para situaciones en las que un estado viola los derechos que tal ordenamiento consagra a favor de los extranjeros en una cierta jurisdicción, es el de la protección diplomática. La protección diplomática implica el inicio de una reclamación internacional por parte del estado cuya nacionalidad tiene el inversor extranjero y sus derechos han sido violados, contra el

20. En los TLC modemos los mecanismos de solución de disputas entre los estados signatarios se encuentran separados de los capitulos de inversiones. 
estado alegadamente ofensor. Presupone, asimismo, que el inversor extranjero ha agotado los remedios internos sin que el daño o agravio haya sido reparado²1. Es decir, el acceso a un remedio internacional presupone: i) intervención necesaria del estado cuyo nacional ha sido afectado; y ii) agotamiento de remedios internos.

Los TBIs, en tanto lex specialis, han transformado dramáticamente el referido escenario al permitir que los inversores administren por sí mismos los derechos allí consagrados, sin que sea necesario el agotamiento de los remedios internos ${ }^{22}$. Esta relación inmediata entre el inversor y el acceso a una jurisdicción para hacer valer los derechos consagrados por el TBI, sin que sean necesarios ni intermediarios ni largas dilaciones y esfuerzos ante las cortes locales, explica la importancia, difusión y utilidad de estos instrumentos en la escena del Derecho Internacional económico contemporáneo. ${ }^{23}$

$\mathrm{El}$ acceso a las jurisdicciones que consagran los TBIs presupone el previo cumplimiento acumulativo de requisitos que pertenecen a tres niveles técnico-legales diferenciados. En primer lugar, las circunstancias subjetivas, objetivas y temporales de la disputa deben ser compatibles con el campo de aplicación del TBI, según se define en cada uno de los instrumentos. En segundo lugar, quien invoque en su beneficio las normas relativas a la solución de controversias entre los inversores y los estados debe dar cumplimiento a los requisitos materiales o procesales adicionales, como por ejemplo, períodos de espera, o formalidades de otra naturaleza, que establecen la mayoría de los TBIs como condicionantes del consentimiento a tales mecanismos jurisdiccio-

21. Ver sobre el punto: "Principles of Pablic International Lam", lan Brownlie; sexta edición, Oxford University Press, p. 472.

22. Este es el caso en la inmensa mayoria de los TBls. Hay algunos TBls, sin embargo, que requieren el uso previo de los tribunales locales por un cierto periodo. Ver articulo 9 (2) del TBt Ecuador - China: "Si el conflicto no puede ser resuelto por medio de negociaciones en un plazo de seis meses, cualquier parte del conflicto tendrd derecho a presentar el mismo al tribunal competente de la Parte Contratanse que acepta la inversion."

23. Nótese, sin embargo, que las primerals generaciones de TBls (década del '60) no establecían un mecanismo de solución de disputas entre inversores y estados. 
nales. Finalmente, los reglamentos o normas que regulan las jurisdicciones a las que un cierto TBI permite acceder, pueden requerir el cumplimiento de pautas adicionales. Por ejemplo, el Convenio CIADI requiere el cumplimiento de ciertas pautas rationae materine, rationae personae y rationae temporis que delimitan la jurisdicción de tal centro de arbitraje. Enfocaremos nuestra atención, en lo sucesivo, en los requisitos mencionados en segundo lugar, es decir, hacia aquellos que hacen a la operación, a los aspectos procedimentales de las cláusulas de solución de controversias entre inversores y estados que incorporan los TBIs.

\subsection{Períodos de espera/negociación}

La inmensa mayoría de los TBIs establecen una primera barrera temporal comúnmente denominada periodo de negocinciones amistosas, periodo de espera o periodo de enfriamiento (cooling off period). El TBI Ecuador - Argentina establece, por ejemplo, que: "Toda controversia relativa a las disposiciones del presente Convenio entre un Inversor de una Parte Contratante y la otra Parte Contratante será, en la medida de lo posible, solucionada por consultas amistosas"24.

La duración de este período y sus puntos de referencia varían tratado por tratado. En la gran mayoría de los TBIs suscritos por Ecuador, este periodo tiene una duración de 6 meses y generalmente opera desde que se notifica la existencia de una disputa en los términos del TBI ${ }^{25}$. En algunos TBIs, se impone el deber

24. Ver TBI Ecuador - Argentina, artículo 9 (1).

25. Ver TBI Ecuador - Canada, anticulo 13 (2): "If a dispute has not ben settled amicably within a period of sir month from the date on which it was initiated, it may be subuitted by the investor to arbitration in accordance with paragraph (4). For the purposes of this paragraph, a dispute is considered to be initiated when the investor of one Contracting Party has delivered notice in writing to the other Contracting Parț alleging that a measute taken or nat taken by the latter Contracting Party' is in breach of this Agreement, and that the investor has incurred loss or damage by reason of, or arising out of, that breach." Hay otros TBls que cstablecen periodos de espera que operan tambien con referencia al momento en que tuvieron lugar los hechos o circunstancias que han dado tugar a la disputa. El TBI Perú - Canadá y el capitulo de inversiones del TLC Pcri - Estados Unidos, por cjemplo, cstablecen periodos de espera que operan acumulativamente y tienen como punto de referencia tanto el momento de los hechos o circunstancias que han gencrado la disputa ( 6 meses) como el momento en cl cual se notifica la intención de someterla a arbitraje ( 3 meses). Ver TBI Peru - Canadá, artículos 24 (1), 25 y 26 (1) (b) y TLC Perí - Estados Unidos, artículos 10.16.2 y 10.16.3. 
de intentar resolver la disputa mediante consultas y negociaciones, pero sin embargo, no se establece un plazo concreto para tales fines ${ }^{26}$.

Es importante señalar que este período previo puede operar de distintas maneras según el TBI que se invoque. En los TBIs que permiten acceder tanto a jurisdicción nacional como internacional, suele operar como un antecedente no sólo de la posibilidad de acudir a arbitraje internacional, sino que también puede operar como un requisito previo a la invocación del TBI ante los tribunales locales ${ }^{27}$. Por su parte, en los TBIs que sólo disponen la posibilidad de acceder a una vía internacional, este requisito opera sólo con relación a esta vía 28 .

Dicho período de espera o negociaciones previo se inicia mediante una notificación al órgano o persona responsable por el manejo de las relaciones exteriores del estado huésped. En el caso del Ecuador sería el Presidente de la República. Es usual que se envíe copia al Ministro de Relaciones Exteriores, al ente que tiene a su cargo la defensa del estado frente a reclamos patrimoniales internacionales ${ }^{29} \mathrm{y}$ al Ministerio, poder o dependencia cuya conducta ha generado la disputa. La mayoría de los TBIs no disponen de formalidad alguna a estos fines, sin perjuicio de lo cual, por razones de prueba y conveniencia, se suele utilizar la forma escrita. En particular, no es necesario cumplir los ritualismos impuestos por la legislación en materia de procedimiento administrativo.

26. Ver entre otros: TBI Ecuador - Bolivia, articulo 9(1): TBI Ecuador - Rumania, articulo 10 (1); TBI Ecuador - Pcrú, artículo 8 (1); TBI Ecuador - El Salvador, articulo 10 (1); TBI Ecuador - Costa Rica, articulo 10 (1).

27. Ver por ejemplo: TBI Ecuador - El Salvador, articulo 10 (2): "Si la controversia no hubiere podido ser solucionada en el terrmino de seis meses, a partir del momento en que fue planteada por una u otra de las Paries, podrá ser sometida, a peditlo del imersionista. - O bien a los Trihunales compefentes de la Parte Contratante en cuyo territorio se rcalizi la insersión. -O bien al arbitraje inter. nacional en las comdiciones descritas en el inciso (3)...".

28. Ver por ejemplo; TBI Ecuador - Repuiblica Dorninicana, articulo 13 (2): "Si estas consultas no permiten solucionar la controversia ch un plazo de scis meses, y si el imversionista implicado lo accpta por escrito, la controversia sera sometida a arbitraje..."

29. En el caso de la República del Ecuador seria la Procuraduria General de la República. 
El tenor de la notificación y sus contenidos también son flexibles. O importante es que la notificación cuente con sustento e información suficiente como para que la parte estatal pueda: i) identificar al inversor y verificar que, al menos prima facie, califica como tal de conformidad con los términos del TBI que invoca; e, ii) identificar los actos y hechos atribuibles al estado que se alegan como violatorios del TBI y las normas de tal instrumento que se consideran violadas. En definitiva, la información tiene que ser lo suficientemente completa como para que el estado pueda tomar una posición y decidir si procede o no, dadas las circunstancias, utilizar recursos en un trámite de negociación o solución amistosa. Hay, sin embargo, TBIs, como, por ejemplo, el TBI Ecuador - España, que requieren que la notificación incluya una información detallada ${ }^{30}$, lo cual supone un grado de precisión mayor que el referido.

Es importante señalar, en este sentido, que, como regla, los TBIs no imponen a los estados una obligación de negociar, y que estos períodos operan fundamentalmente en su beneficio como una ventana temporal que implica una oportunidad para intentar desactivar la disputa. Cumplido el plazo, los inversores tienen el derecho, pero no la obligación, de iniciar un trámite jurisdiccional al amparo del TBI, pudiendo administrar tal derecho como mejor les parezca. En este último sentido es importante señalar que, como regla, los TBIs no disponen de plazos de caducidad o prescripción que limiten la posibilidad de acceder a los remedios jurisdiccionales que consagran ${ }^{31}$. Sin embargo, algunos TBIs como, por ejemplo, el TBI Ecuador - Canadá32 disponen de

30. Ver TBI Ecuador - España, articulo II (1) "Toda contronersia relativa a las inversiones que surja cutre una de las Partes Contratantes y un inversionista de la otra Parte Contratante respucto a cuestiones reguladas por el presente dcuerdo scrá notificada, por escrito, incluyendo una informacioin detallada por el inversionista a la Parte Contratante receptora de la inversien... ".

31. Debe notarse, sin embargo, que en algunos casos un derecho a un determinado activo que no ha sido cjercido en tiempo y forma ante los tribunales toeales podría dejar de calificar como una inversión a los fines del campo de aplicación de un TBI.

32. Ver TB1 Ecuador - Canadá, articulo 13 (3): "An investor may submit a dispute as referred to in paragraph (l) to arbitration in accordance with paragraph (4) only if ....(d) not more than three years have elapsed from the date on which the investor first acyuired or should have first acquired knowledge of the alleged breach and knowledge that the investor has incurred loss or damage". 
un plazo de caducidad de tres años contados desde que se tuvo o debió haber tenido conocimiento de la existencia de una violación de tales instrumentos y de la existencia de pérdidas o daños como consecuencia de ésta.

En varias ocasiones los inversores han obviado este período previo y han pretendido activar los mecanismos jurisdiccionales que disponen los TBIs sin cumplir con esta etapa. Los tribunales han reaccionado de distinta manera frente a tal hipótesis ${ }^{33}$. En algunos casos, han optado por calificar como meramente procesal a tal período y permitir la continuidad del procedimiento pese al incumplimiento ${ }^{34}$. En otro caso, aunque se permitió la continuidad del procedimiento, se obligó al inversor a cargar con las costas del incidente procesal derivado de su accionar anticipado ${ }^{35}$. Por su parte, una tercera posición ha sido la de considerar que la falta de cumplimiento de esta instancia previa afectaba la jurisdicción del tribunal ${ }^{36}$. En otro caso, como cuarta alternativa, un tribunal arbitral decidió suspender el procedimiento hasta que se cumpla el plazo de 6 meses dispuesto por el tratado aplicable ${ }^{37}$. Es de notar que la Secretaría del CIADI también ha dilatado el registro de casos en lo que se verificaba una falta de cumplimiento de estos requisitos temporales.

33. Ver "Travelling the BIT Route, of Waiting Periods, Umbrella Clauses and Forks in the Road", C. Schreuer; Journal of World Investment \& Trade. Volume 5. 2004. p. 235.

34. Ver Remald S. Lumder versus Reptiblica Checa, [laudo del 3 de septiembre de 2001 (NAFTA)]: y, SGS Secièté Ginérale de Surveillance S.A. versus República Islämica de Pakistán. [decisión sobre Jurisdicción de 6 de Agosto de 2003. Casu ClADI ARB/01/13]. Ambas decisiones están publicadas en hitp://ita.law.uvic.ca.

35. Ver Ethyl Corporation versus Canada, [decisión sobre Jurisdicción del 24 de junio de 1998 (NAFTA)], publicada en http://ita.law.uvic.ca.

36. Ver Goetz and olhers versus Burundi, [laudo (de común acuerdo) de 13 de febrero de 1999, Caso CIADI ARB/95/3]. Es menesier notar, sin cmbargo, que el TBI aplicable en este caso requeria que durante el periodo en consideración también se verificara una instancia de negociación entre las partes contratantes. Otras dos decisiones arbitrales han scĩalado, como obiter dicta, que el incumplimiento de este plazo previo puede afectar la jurisdicción del tribunal. Ver en este sentido, obiter dicta: Enron and Ponderosa Assets versus Repuhlica Argentina, [decisiön sobre Jurisdicción de 14 de enero de 2004, Caso CIADI ARB/01/3]; y, Generation Ukraine Inc. versus Ucrania, [laudo de 16 de septiembre de 2003, Caso CIADI ARB/00/9]. Ver tambièn en este sentido Burlington Resources Inc. y otros versus Repuihlica del Ecuador y Empresa Estatal Petróleos del Ecuador (PetroEcuador), [Decisión sobre Jurisdieción del 10 de Junio de 2010, Caso CIADI No. ARB/08/5].

37. Ver Nestern NIS Enterprise Fumd versus Ucrania, [orden Procesal de 16 de marzo de 2006, Caso CIADI ARB/04/2]. 
Puede suceder muchas veces que un estado dicte una secuencia de medidas o incurra en actos que afectan a un inversor en distintos momentos. La pregunta, en estos casos, es si el inversor tiene que repetir el procedimiento con relación a cada una de ellas. La respuesta no es simple y dependerá en gran medida de las circunstancias, pero puede decirse, de manera general, que si existe un nexo de identidad o continuidad entre las disputas que se generan como consecuencia de tales medidas, no será necesario repetir el procedimiento. En tal escenario, las nuevas circunstancias se incorporarán al marco de la disputa existente mediante una notificación o al momento de iniciarse el procedimiento, o estando éste en trámite, como un hecho nuevo, o una demanda subordinada, ${ }^{38}$ según lo establezca el reglamento procesal aplicable.

El tema se planteó, por ejemplo, en un caso contra Argentina, en el cual un inversor comunicó la existencia de una alegada violación de un TBI como consecuencia de ciertas determinaciones fiscales iniciadas por distintas provincias contra un inversor. Luego, otras provincias hicieron lo mismo. La cuestión era si la disputa denunciada al amparo del TBI aplicable con relación a los actos de una de las provincias cubría también a las posteriores medidas idénticas tomadas por las otras. El tribunal actuante concluyó que no hacía falta agotar un nuevo período de espera, dada la identidad y relación entre unas y otras medidas ${ }^{39}$.

En definitiva, en todo caso, interpretar rectamente el efecto de estos períodos de espera sobre los derechos procesales que consagran los TBI requiere preservar el delicado balance entre i) mantener la vigencia de las estipulaciones específicas negocia-

38. Ver regla 40 de las Reglas de Arbitraje del CIADL. En cl caso CMS Gas Transmission Company versus Repuiblica Argentina.[decisión sobre Jurisdicción de 17 de julio de 2003, Caso CIADI ARB $3 / 01 / 8]$, se usó esta vía para ampliar el tipo de medidas sobre las cuales se pronunció cl tribunal. Sin cmbargo, es cuestionable que en tal caso existicra identidad y continuidad entre unas y otras medidas.

39. Ver por cjemplo, Enron and Ponderusa Assets versus Repühlica drgentina. [decistón sobre Jurisdicción de 14 de enero de 2004, Caso CIADI ARB/01/3]. 
das por las partes, evitando que se transformen en letra muerta; $y$, ii) evitar caer en formalismos excesivos frente a hipótesis en las que el Estado tuvo una genuina oportunidad de ponderar qué actitud asumir frente a la disputa.

\subsection{Opciones jurisdiccionales}

Los TBIs estructuran las opciones jurisdiccionales disponibles para quien califica como inversor de manera variada. En general, existen cuatro modelos que se describen a continuación. Los TBIs suscritos por Ecuador siguen fundamentalmente los modelos descritos en ii), iii) y iv).

i) Sistema escalonado: el inversor debe utilizar los remedios locales por un cierto período como requisito previo al uso de la vía arbitral internacional, salvo acuerdo de partes. En los TBIs suscritos por Ecuador, no se encuentra presente este tipo de sistema ${ }^{40}$.

ii) Sistema alternativo o de opción de vias (fork in the road): el inversor cuenta con la opción de acceder a la jurisdicción local o a un arbitraje internacional. Algunos de los TBIs disponen específicamente que tal elección es definitiva. Un ejemplo de éste sistema lo constituye el TBI Ecuador - Argentina, el cual dispone lo siguiente:

"Si la controversia no hubiere podido ser solucionada en el término de seis meses a partir del momento en que hubiera

40. Un cjemplo del sistema escalonado es el TBI Peru - Alemania, articulo 10 (2) y (3): "(2) Si una contrviersia, en el sentido del parrafo 1. no pudiera ser resuelta dentro del plazo de seis meses, contada desde la fecha en que una de las partes en la controsersia la haya promovido, será somelida a petición de una de ellas a los tribunales competentes de ta parte contratante en cano territorio se realizi la inversion. (3) La controversia podra ser sometida a un tribumal arbitral internacional en ctualquicra de las circunstancias sigutentes:(a) A peticiön de una de las partes de la controversia, cuando no exista una decision sobre el fondo después de transcurridos dieciocho meses, contados a partir de la iniciaciën del proceso judicial previsto por el apartado 2 de este articulo, o cuando exista tal decision pero la controversia subsista entre las partes. (b) Cuando ambas partes en la controversia asi lo hayzan comenido". 
Parlicularidades del acceso al Arbitraje Internacional en los Traiados Bilaterales de hacrsión suscritos por la República del Ecuador

sido planteada por una u otra de las Partes, podrá ser sometida, a pedido del inversor:

- o bien a los tribunales competentes de la Parte Contratante en cuyo territorio se realizó la inversión;

- o bien al arbitraje internacional en las condiciones descritas en el párrafo (3).

Una vez que un inversor haya sometido la controversia a las jurisdicciones de la Parte Contratante implicada o al arbitraje internacional, la elección de uno u otro de esos procedimientos será definitiva" 41 .

iii) Sistema de elección de vías con vuelta en $U$ ( U turn system): el inversor puede utilizar los remedios locales sin perder la posibilidad de acceder a arbitraje internacional, pero se requiere una previa renuncia a cualquier uso de la jurisdicción local, +2 como requisito previo al inicio de un arbitraje internacional. El TBI Ecuador - Canada ${ }^{43}$ sigue este sistema.

iv) Sistema de acceso directo: el inversor accede directamente a alguna variante de arbitraje internacional. El TBI Ecuador Suecia y el TBI Ecuador - República Dominicana constituyen ejemplos en este sentido. El último de los instrumentos mencionados establece el acceso directo en los siguientes términos:

"Si estas consultas no permiten solucionar la controversia en un plazo de seis meses, y si el inversionista implicado lo acepta por escrito, la controversia será sometida a arbitraje al Centro Internacional de Arreglo de Diferencias Relativas a Inversiones (CIADI) ..." +4 .

41. Ver TBI Ecuador - Argentina, articulo 9 (2).

42. Ello incluye reclamos en sede local por violación del TBI o el capitulo de inversión. Ver Waste Afanagement Inc. Iersus Aféxico (NAFTA), [laudo sobre Jurisdicciỏn de 2 de junio de 2000, Caso CIADI ARB (AF)/98/2].

43. Ver TBI Ecuador - Canada, articulo 13.3 (b): "An Insestor may submit a dispute as referned to in paragraph (I) to arbitration in accuriance with paragraph (4) only if ...h) the investor has watived its right to initiate or continue any other proceedings in relation to the measure that is alleged to be in breach of this Agreement before the courts or tribunals of the Contracting Party concerned or in a dispute setllement procedure of any kind".

44. Ver: TBI Ecuador - República Dominicana, articulo 13 (2). Ver también TBI Ecuador - Suecia, artículo 8 (2 y (3): "Si cualquier controversia no ha podido ser resuelia en un plazo de seis meses des- 
De los sistemas referidos el que presenta mayores inconvenientes prácticos e incertidumbres es el alternativo o de opción de víns, especialmente cuando el TBI dispone expresamente que el acudir a los tribunales competentes locales puede tener efectos preclusivos sobre la posibilidad de acceder posteriormente a un arbitraje internacional. La gran duda en cuanto a este punto es qué tipo de uso de los remedios jurisdiccionales locales tiene como consecuencia una eventual pérdida de la posibilidad de acceder posteriormente a un arbitraje internacional. $\mathrm{O}$, desde otra perspectiva, cómo se puede hacer para preservar los derechos que se tienen bajo el ordenamiento ecuatoriano y, por ejemplo, no consentir en la vía administrativa un accionar que de otra manera devendría firme, sin perder la posibilidad de acudir, si fuera necesario, a una posterior instancia internacional.

La respuesta a los interrogantes conexos formulados en el párrafo anterior varían TBI por TBI. Como primer dato orientador, es importante distinguir sobre qué bases está articulada la opción de vías que establece el TBI que se pretende invocar. Hay algunos TBIs cuyas cláusulas sobre la materia se refieren a toda controversia relativa a las disposiciones del presente Convenio ${ }^{45}$. En este caso, utilizar los tribunales locales para planteos de derecho local en los que se formulan planteos o peticiones que no tienen base en el TBI, no implicaría sacrificar el posterior derecho de acudir a un arbitraje internacional, aunque haya superposición total o parcial desde el punto de vista subjetivo o en cuanto a las circunstancias fácticas que se ventilan en ambas instancias. Esta conclusión está avalada por numerosos pronunciamientos arbitrales, según los cuales es necesario que exista; i) identidad sub-

pués de la fecha en que la contmversia fue planteada por el imcrsionista a través de la notificacion escrita a la Parte Contratante, cada tma de las Partes Contratantes consiente por el presente a someter la solucion de la contmoversia, a seleccion del imersionista, a resolucioin de un arbitraje internacional en unos de los siguientes foros: i) el Centro Internacional para el Arreglo de Disputas por Inversiones (ClADl) para el arreglo por conciliacion o arhitraje .... o ii) un tribual ad-hoc constimido bajo las Reglas para el Arbitraje de la Comisión de las Naciones Unidas sobre Derecho Mercantil luternacional (UNCITRAL)...Si las partes de dicha controversia tienen opiniones diferentes sobre si la conciliación o el arbitraje es el método de soluciỏn más apropiado, el intersionista tendra derecho de decidir".

45. Ver TBI Ecuador - Argentina, artículo 9 (1). 
jetiva; e, ii) identidad causal en cuanto al sustento legal del reclamots.

Hay otros TBIs, sin embargo, cuyas cláusulas relativas a disputas entre inversores y estados se refieren, por ejemplo, a las controversins que surgieren entre una de las Partes Contratantes y un inversionista de la otra Parte Contratante en relación con las inversiones realizadas de conformidad con el presente Convenio. ${ }^{47}$ En este caso, en la medida en que acudir a una corte local implica una controversia en relación con una inversión, existe el riesgo de que se considere que tal accionar ha implicado un sacrificio de la vía internacional, especialmente si hay identidad de partes..$^{48}$

En todo caso, debe notarse que cierta jurisprudencia i) distingue a estos fines entre medidas activas y medidas defensivas, considerándose que éstas últimas no constituyen una elección de una vía procesal de conformidad con los $\mathrm{TBIs}^{49} \mathrm{y}$ ii) ha asumido una posición restrictiva en cuanto a la posibilidad de que la articulación de reclamos en la vía administrativa constituya un sometimiento de una disputa a un tribunal competente, siendo este el lenguaje que usan la mayoría de los TBIs. ${ }^{5 !}$

El punto central, en lo que concierne a la operación de estas cláusulas, es tener en mente que no fueron concebidas para colo-

46. Ver, entre otros: Alex Genin, Eastern Credit Limited, Inc., and A.S. Bultoi versus República de Estonta, [laudo de 5 de abril de 2002, Caso ClADI ARB/99/2]; Roland S. Lander versus República Checa, [laudo de 3 de septiembre de 2001, Caso CNUDMI]; Middle East Cement Shipping and Handling S.A. versus Egipto, [laudo del 12 de abril de 2002, Caso CIADI ARB/99/6]; CMS Gas Transmission Company versus República Aryentina, [decisión sobre Jurisdicción de 17 de julio de 2003. Caso CIADI ARB/01/8]; Azurix Corp. versus República Argentina. [decisión sobre Jurisdicción de 8 de diciembre de 2003, Caso CLADI ARB/01/12]; y, Enron and Pondervsa Assets versus Republica Argentina, [decisión sobre Jurisdicción de 14 de cnero de 2004. Caso CIADI ARB/01/3].

47. Ver TBI Ecuador - Peru, articulo 8 (1).

48. Ver en este sentido Compañia de Aguas del Aconquija S.A. and Vivendi Universal versus Repuiblica Argentina, [decisión sobre Nulidad de 3 de julio del 2002, Caso ClADl ARB/97/3].

49. Ver CMS Gas Transmission Company versus Repriblica Argentina, [dccisión sobre Jurisdicción de 17 de julio de 2003, Caso ClADI ARB/01/8]; Enron and Ponderosa issets versus Repuiblica Argentina. [decisión sobre Jurisdicción de 14 de enero de 2004, Caso CIADI ARB/01/3]; Azurix Corp. versus Repriblica Argentina, [decisión sobre Jurisdicción de 8 de diciembre de 2003, Caso CIADI ARB/01/12].

50. Ver Azurix Corp. versus Repuiblica Argentina, [decisión sobre Jurisdieción de 8 de diciembre de 2003, Caso ClADI ARB/0I/12]. 
Iguacio Sutre:

car a los inversores en la incómoda disyuntiva de elegir entre sacrificar derechos en la vía interna o en la vía internacional en el contexto de los breves plazos legales del derecho local, sino para evitar abusos y especulaciones procesales reñidas con la buena fe.

\subsection{Jurisdicciones disponibles}

Los TBIs varian en cuanto al menú de opciones jurisdiccionales disponibles para solucionar disputas entre inversores y estados. Como se describe en el punto anterior, algunos TBIs permiten elegir entre los tribunales locales y el arbitraje internacional, mientras que otros solo habilitan esta última vía jurisdiccional. A su vez, algunos TBIs permiten una sola opción a estos fines, ${ }^{51}$ mientras que otros permiten elegir entre distintos tipos de arbitraje internacional, como arbitraje de conformidad con el Convenio CIADI y/o de conformidad con el denominado Mecanismo Complementario del CIADI52, o arbitraje bajo las reglas CNUDMI. Asimismo, hay ciertos TBIs firmados por Ecuador que se refieren exclusivamente a la posibilidad de utilizar los servicios que en materia de arbitraje brinda el CIADI, mientras que otros también incluyen, generalmente a criterio del inversor, la posibilidad de utilizar los servicios de conciliación que brinda tal entidad. ${ }^{53}$

La mayoría de los inversores que cuentan con la posibilidad de acceder a un arbitraje CIADI eligen esta vía dada las extraordinarias garantías de neutralidad y eficacia que brinda ${ }^{54}$. A los

51. Por ejemplo, el TBI Ecuador - Chile contempla exclusivamente la posibilidad de arbitrar las disputas ante el ClADI (articulo 10 (3)). El TBI Ecuador-China sólo permitc utilizar un arbitraje ad-hoc en el que la autoridad nominadora es el Secretario del ClADI (articulo 9 (4)).

52. Ver TBI Ecuador - Canada, articulo 13 (4) (b): "The dispute may: at election of the imvestor concerned. be submincd to arbitration under:...b) the ddditional Facility Rules of ICSID. provided that cither the disputing Contracting Party or the Contracting Party of the investor, but not both, is a party to the ICSID Convention;...".

53. Ver, por cjemplo el TBI Eccuador - Suecia, el cual dispone en su articulo 8 (2) (ii) in fine que "...Si las partes de dicha controversia sienen opiniones diferentes sobre si la conciliacion o el arbitrajc es el método de solucion más apropiado, el inversionista tendra derecho de decidir". Ver también el TBI Ecuador - Francia, (articulo 9) y el TBI Ecuador - Gran Bretaña, (articulo 8). 
fines de promover un marco de neutralidad, el Convenio CIADI: i) establece un conjunto de privilegios e inmunidades en beneficio del Centro, los procedimientos y sus participantes ${ }^{55} \mathrm{y}$, ii) excluye la participación de tribunales estatales en la revisión de los laudos arbitrales ${ }^{5}$. A los fines de brindarle eficacia a disputas que suelen tener un alto grado de politización, el Convenio dispone que los laudos emitidos bajo su órbita sean de reconocimiento y ejecución automática en todos los estados contratantes, como si fueran una sentencia judicial local definitiva ${ }^{57}$. No es necesario ni un trámite de exequátur ni ningún otro procedimiento de nacionalización del laudo o sentencia extranjera de conformidad con el Derecho local o con otros tratados internacionales sobre la materia. Lo dicho, sin perjuicio de que el procedimiento de ejecución se rige por el Derecho local, con lo que ello implica en materia de pautas de inmunidad de ejecución del estado $^{58}$. Todo el esquema se encuentra reforzado por el hecho de que el incumplimiento del Convenio CIADI por parte de un estado contratante genera responsabilidad internacional y permite a los otros estados contratantes iniciar acciones ante la Corte Internacional de Justicia contra el incumplidor ${ }^{59}$.

Como contrapartida de estas ventajas, el Convenio CIADI establece requisitos rationae personae y rationae materiae que restringen el acceso a la jurisdicción de tal Centro en razón del tipo de activos y de la nacionalidad y naturaleza de las partes. ${ }^{60} \mathrm{De}$ conformidad con el Convenio CIADI, i) la disputa debe surgir directamente de una inversión en los términos del Convenio y, asimismo, ii) una de las partes debe ser un nacional de un estado contratante $y$, la otra, un estado contratante $o$, en ciertas cir-

54. Son raros los casos en los que una parte que puede acudir a arbitraje bajo el convenio CIADI elija otra alternativa. La mayoria de los casos ante otros mecanismos arbitrales se explica porque, o bien el arbitraje bajo el Convenio CIADI no estaba disponible, o bien la disputa no cumplia con los requisitos rationac personac y rationac materiac que tal instrumento cstablece.

55. Ver Convenio CIADl, articulos 19 al 24.

56. Ibid. articulos 49 al 52

57. Ibid, articulo 54 .

58. Ibid articulo 55 .

59. Ibid. articulo 64 .

60. Ibid. articulo 25 . 
cunstancias, una de sus subdivisiones políticas u organismos públicos ${ }^{61}$. Tales requisitos no están presentes ni en el caso de arbitrajes bajo las reglas CNUDMI ni en el caso de arbitrajes bajo el denominado Mecanismo Complementario del CIADI62.

La denuncia por parte de Ecuador del Convenio CIADI puede tener efectos sobre disputas cuyo sometimiento a tal mecanismo de solución de controversias pueda reputarse como no consentido al momento en que tal denuncia se hizo efectiva. ${ }^{63}$ La solución para cada caso dependerá del momento de la ocurrencia de la disputa y las acciones que haya tomado el inversor para que tal consentimiento se pueda tener por configurado, y del lenguaje específico del TBI que se invoque.

\subsection{Extensión material de la cláusula de solución de controversias}

Como regla general, las disposiciones de los TBIs en materia de disputas inversor-estado comprenden la posibilidad de alegar cualquier violación de las garantías específicas que consagra el TBI que se invoque. Esto es particularmente claro en el caso de TBIs que identifican las disputas que pueden plantearse bajo sus términos mediante una referencia al ordenamiento jurídico que debe invocarse en sustento del reclamo. Así, por ejemplo, el TBI Ecuador - Argentina se refiere a toda controversia relativa a las disposiciones del presente Convenio. ${ }^{64}$ En este caso, es indubitable que:

61. Ver "Introduccion a los requisitos rationae materiae y rationac personae del arbitraje hajo el Convenio ClADr'. C. Ignacio Suarez Anzorena, Revista Peruana de Arbitrajc, Volumen 2. 2006. pp. 252 y siguientes.

62. El Mecanismo Complementario del CIADI se aplica a disputas entre estados e inversores extranjeros que sólo cumplen parcialmente con los requisitos jurisdiccionales que establece el Convenio CIADI (id est, uno de las partes no es nacional de un estado contratante o la disputa no surge directamente de una inversión). Bajo el Mecanismo Compiementario, el ClADI presta servicios de administración de procedimientos de arbitraje, procedimientos de conciliación y procedimientos de comprobación de hechos. Los procedimientos de solución de disputas bajo el Mecanismo Complementario no están amparados por el Convenio CIADI, y por ello no gozan de las inmunidades, privilegios y ventajas que alli se consagran.

63. Ver Convenio CIADI, articulo 72. La denuncia es efectiva desde el 7 de enero de 2010, es decir, 6 meses luego de la notificación., tal como lo dispone el articulo 71 del Convenio CIADL. 
i) sólo puede alegarse una violación del TBI; y, ii) toda otra alegación o reclamo está excluida del campo de aplicación de la cláusula.

Más complejo es el panorama en lo que respecta a los TBIs que definen el campo de aplicación de sus disposiciones relativas a disputas inversor-estado con un lenguaje que enfocado en los activos protegidos, es decir, las inversiones, y no en los derechos que se invocan. El TBI Ecuador - Perú, por ejemplo, hace a referencia a disputns relacionadas con las inversiones las controversias que surgieren entre una de las Partes Contratantes y un inversionista de la otra Parte Contratante en relación con las inversiones realizadas de conformidad con el presente Convenio 656 6. La pregunta es si esta definición comprende, por ejemplo, una alegación de violación de contrato, que no necesariamente implica violación del TBI aplicable, pero claramente califica como una inversión realizada de conformidad con el Convenio. La jurisprudencia sobre el punto es inconsistente, aunque desde el punto de vista práctico puede decirse que los tribunales arbitrales constituidos bajo TBIs han sido reacios a habilitar este tipo de reclamos. ${ }^{67}$

Distinto es el caso de los TBIs que expresamente tienen una disposición sobre conversión de jurisdicción. El TLC Perú-

64. Ver TBI Ecuador - Argentina, articulo 9 (1).

65. Ver TBI Ecuador - Peri, anticulo 8 (1). Ver también TB1 Ecuador - Francia, articulo 9.

66. Hay cláusulas que restringen más aủn el campo de apiicación de los TBls, Asi, por ejemplo, el TBI Ecuador - China, cn su ariculo 9 (3): "Si un conflicto relacionado con el monto de compensación por expropiación no puede ser nesuclio en un plazo.... En un caso de TBI entre Bélgica y Rusia, Berschader versus Rusia, [laudo de 21 de abril de 2006, Caso CCE No. 080/2004], el Tribunal decidió que no podia extender su jurisulieción más allá de la consideración del monto de la expropiación. Por el contrario, en otros casos como Tza Yap Sham. versus Repuiblica del Peri. [decisión sobre Jurisdicción de 19 de junio de 2009, Caso CIADI ARB/07/6], la jurisprudencia arbitral ha reconocido que su aplicación alcanza todos los aspectos de la expropiación, no sólo el monto sino también la determinación de la expropiación misma. En el mismo scntido, RosInvest UK Lud versis Rusia. [decisión sobre Jurisdicción de Octubre de 2007, Caso CCE].

67. Ver, a favor de la posibilidad de plantear reclamos contractuales: SGS Société Gíniralc de Surveillance S.A. versus Filipinas, [decisión sobre Jurisuicción de 29 de enero de 2004, Caso CIADI ARB/02/6]; y, aunque de manera mús difusa, Salini Construtorri S.p.A. and Itulstrade S.p.A. verssus Afarritecus, [decisión sobre Jurisdicción de 23 de julio de 2001, Caso ClADI ARB/ 00/4]. En contra, ver: SGS Socięté Gènerale de Surveillance S.A. versus Pukistán, [decisión sobre Jurisdicción de 6 de agosto de 2003, Caso CIADI ARB/01/13]. Cabe hacer notar que, en el primero de los casos, el tribunal decidió que tenia jurisdicción pero ordenó suspender el procedimiento sobre el fondo hasta que se resuclva el reclamo contractual en el fucro pactado en el contrato. 
Estados Unidos, por ejemplo, permite que los inversores inicien un caso no sólamente basado en las violaciones de las protecciones sustanciales del TBI sino que también pueden invocar la violación de una autorización de inversión o de un acuerdo de inversión. ${ }^{68}$ El efecto concreto de estas cláusulas de conversión de jurisdicción es permitir a los inversores alegar violaciones contractuales ${ }^{69}$ ante el foro del TBI o del TLC independientemente del sistema de solución de controversias pactado contractualmente y sin que sea necesario alegar una violación de los derechos sustantivos establecidos por el tratado. El TBI EcuadorCanadá ${ }^{70}$ por ejemplo, aunque tiene alcances más limitados que el TLC mencionado, permite expresamente que se utilice el mecanismo de solución de disputas de tal instrumento para solucionar disputas en materia de impuestos que implican una alegación de que se ha violado un acuerdo entre el gobierno y el inversor sobre tal materia.

Hay, sin embargo, algunos TBIs que restringen las materias sobre las que pueden versar tales disputas.

68. Ver TLC Peri - Estados Unidos, artículos 10.16-1-(a) (i) (B), 10.16-1-(a) (i) (C), 10.16-1-(b) (i) (B), 10.16-1-(b) (i) (B), definiciones de acuerdo de inversión y autorización de inversión cn art. 10.28, y exclusiones con relación a ciertos tipos de convenios de estabilidad juridica en Anexo 10- $\mathrm{H}$.

69. No debe confundirse esta situación con la alegación de que un estado ha violado una clảusula de las denominadas paraguas que contienen ciertos TBIs y que, según cierta jurisprudencia, permite alegar que una violación de un contrato estatal constituye, a la vez, una violación de un TBI. Un ejemplo de cláusula paraguas puede encontrarse en el articulo 7 (2) del TBI Ecuador - Alcmania, segùn el cual: "Cada Parte Contratante cumplira cualquier otrs compromiso que haja contraido con relación a las inversiones de capital de nacionales o sociedades de la otra Parte Contratante en su territorio".

70. Ver TBI Ecuador - Canadả, articulo 12 (3): "Suhject to paragraph (2) a claim by an imvestor that a tax measure of a Contracting Party is in breach of an agreement between the central government authorities of a Contracting Party and the investor concerning an investment shall be considered a claim for breuch of this Agretment unless the taxation authorities of the Contracting Parties, no later than six months afier being notificd of the claim by the investor, jointly determine that the measure does not contravene such agreenent". Ver también el TBI Ecuador - República Dominicana, articulo II (2): "Toda reclamación de un imersionista de que una medida fiscal de una de las Partes Contratantes viola el acuerlo entre las autoridades del gobierno centrai de tunu Parte Contratante y el inversionista con respecto a una inversión, se considerará reclamación por violación de este Acserdo a menos que las autoridades fiscales de las Partes Contratantes determinen conjuntamente, en un plazo no major de seis meses después de ser notificado de la reclamaciön por el inversio. nista, que la medida no contraviene tal acuerdo." 
Particularidades del acceso al Arbitraje Internacional en los Tratados Bilatcrates de Inversión suscritos por la Repuiblica del Ecuador

EI TBI Ecuador - USA, por ejemplo, dispone que sus normas serán de aplicación en materia tributaria sólo en casos de expropiación, transferencias y de cumplimiento de los términos de un acuerdo o autorización de inversión. ${ }^{71}$ Disposiciones de naturaleza comparable también están presentes en el TBI Ecuador Canadá y en el TBI Ecuador - República Dominicana.

El TBI Ecuador - China, por su parte, tiene un lenguaje que sugiere que sólo puede acudirse a arbitraje en el supuesto de $u n$ conflicto relacionado con el monto de compensación por expropiación. ${ }^{72}$ En el punto siguiente se analiza en que medida estas limitaciones pueden ser superadas mediante la invocación de las cláusulas de nación más favorecida.

\section{Operación de las Cláusulas de nación MÁS FAVORECIDA EN MATERIA PROCESAL}

Uno de los puntos más álgidos del Derecho Internacional de las inversiones contemporáneas se relaciona con la posibilidad, o no, de invocar las cláusulas de nación más favorecida a los fines de suplantar o modificar las normas aplicables a las disputas inversor-estado que dispone un cierto TBI. En los párrafos

71. Ver TBI Ecuador - Estados Unidos, articulo 10 (2): "No obstante las disposiciones del prescute Tratado, especialmente de les Articules $V /$ y VII det mismo, se aplicarain a cuestiomes tributarias solamente con respecto a: a) La expropiacion. de conformidad con el Articulo III: b) Las transferencias de conformidad con el Articulo IV. o c) La obsernancia y cl cumplimicnto de les tírminos de un acuerdo o autorizacioin en matcria de inversion, tal como se menciond en el inciso a) a el inciso b). en la medida ch que estén sujectes a las disposiciones sobre la sohciòn de diferencias de un Comvenio para critar la doble imposicion tributaria concertado entre las dos Partes, o que se hajan suscitodo de conformidad con dichas disposiciones y no se hay an resucho en un plazo razonable". Esta disposición fue objeto de controversia en el primero de los casos iniciados por Occidental Peirolezm contra Ecuador. Ver tambićn el TB1 Ecusdor - Canadá, articulo 12 (1), el cual fue analizado en el caso Encana Corporation 1: Ecuator, citado más arriba.

72. Ver TBI Ecuador - China, articulo 9 (3). Este tipo de disposiciones ha sido objeto de un tratamiento jurisprudencial inconsistente. En Berschuder v: Russian Federation, [laudo de 21 de abril de 2006. Caso CCE No. 080/2004], el Tribunal decidió que no podia exıender su jurisdicción mas alla de la consideración del monlo de la expropiación. Por el contrario, en otros casos como Ta Yap Shum. versus Repriblicu del Perii, [decisión sobre Jurisdicción de 19 de junio de 2009, Caso CIADI ARB/07/6], Roslmesı UK Lud versus Rusia, [decisión sobre Jurisdicción de Octubre de 2007, Caso CCE], se decidió que esta cláusula se extiende no sólo el monto sino también la determinación de la existencia de la expropiación misma. 
sucesivos se delinean, aunque sea someramente, los alcances de una polémica que, si bien relevante, excede en su tratamiento pormenorizado a la temática de esta contribución.

Las cláusulas de nación más favorecidas son disposiciones que implican el compromiso por parte de los estados contratantes de darle a los beneficiarios de sus términos (en este caso, los inversores bajo un cierto TBI) un tratamiento no menos favorable que el que se les da a otros nacionales en lo que concierne a ciertas materias.

La operatividad de estas cláusulas con relación a los mecanismos de solución de controversias para disputas inversor-estado de los TBIs presupone: i) que un cierto TBI contiene una cláusula de este tipo; ii) que otro TBI le brinda a otros inversores ventajas en materia del sistema de solución de disputas entre inversores y estados; $y$, iii) que el lenguaje de la cláusula permite inferir que tal materia está entre aquellas respecto de las cuales la cláusula es operativa. La gran discordancia que actualmente se verifica en la jurisprudencia de los tribunales arbitrales se relaciona con este último aspecto.

Las aguas están divididas, fundamentalmente, entre i) aquellas decisiones que consideran que dada la importancia del arbitraje internacional en el campo de la protección de inversiones debe construirse liberalmente los términos de las cláusulas de nación más favorecida, en cuanto a su aplicación al sistema de solución de controversias inversor-estado ${ }^{73} \mathrm{y}$ ii) aquellas decisiones que consideran que las cuestiones procesales, como regla, sólo forman parte del campo operativo de tales cláusulas cuando ello puede inferirse claramente de sus términos ${ }^{74}$.

73. Ver Maffezini versus Reino de Españã, [decisión sobre Jurisdicción de 25 de enero de 2000, Caso CIADI ARB/97/7]; Sienchs versus Argentinu, [decisión sobre Jurisdicción de 3 de agosto de 2004, Caso CIADI ARB/02/8]; National Grid PLC versus Repriblica de Argentina, [decisión sobre Jurisdicción de 20 de junio de 2006, Caso CNUDMI/BIT, párrafo 82]; Gus Nutural SDG S.A. versus Repuiblica de Argentina, [laudo de 17 de junio de 2005, Caso ClADl arb/03/10]: Agnas Provinciales de Santa Fe S.A. Suez, Socicdad General de Aguas de Barcelona, S.A. e Interdguas Senvicios Integrales del Agita, S.A. versus Repuiblica de Argentina, [decisión sobre Jurisdicción de 16 de mayo de 2006, Caso CIADI ARB/03/17]; Buyindir versus Pakistán, [decisión sobre Jurisưicción de 14 de 
Es importante tener en cuenta que las cláusulas de nación más favorecida varían en su texto y estructura, por lo que no son aconsejables las generalizaciones. Este fue justamente el problema de algunas de las primeras decisiones que fueron permisivas sobre esta materia ${ }^{75}$, cuya cuestionable factura técnica generó numerosas polémicas. No hay ninguna razón de principios para que una cláusula de nación más favorecida no se aplique a los aspectos procesales del TBI, si ello puede inferirse de sus términos. En definitiva, el tema es meramente hermenéutico y debe resolverse en función de la letra y estructura de cada tratado en particular.

\section{CONSIDERACIONES FinAles}

El arbitraje entre inversores y estados con base en los TBIs ha entrado en su adolescencia desde el punto de vista legal. Crece a una velocidad que dificulta una visión retrospectiva crítica de los acontecimientos y de los problemas sistémicos que su aplicación plantea. Como buen adolescente, tiene facetas inconsistentes e inciertas, como lo demuestra la jurisprudencia que se cita en este trabajo. Asimismo, plantea delicados dilemas sistémicos como consecuencia de la combinación entre ofertas de jurisdicción internacional abiertas a una generalidad de individuos y mecanismos arbitrales descentralizados con tribunales ad

noviembre de 2005, Caso ClADI ARB/03/29; BG Group PLC versus República Argentina, [laudo de 24 de diciembre de 2007. Caso CNUDMI]. En este último caso, el Tribunal adhirió a la interpretación amplia de la clảusula de nación más favorecida. pero no ahondó en su análisis.

74. Ver Salini Construtorri S p.A and Italstrade versus Joriania; [decisión sobre Jurisdicción del 9 de novicmbre de 2004, Caso ClADI ARB/02/13]; Plama Consortium Limited verstus Bulgaria; [decisión sobre Jurisdicción de 8 de febrero de 2005, Caso ClADl ARB/03/24]; Telenor Mobile Communications A.S. versus Hungria, [laudo de 13 de septiembre de 2006, Caso CIADI ARB/04/15]; Tza Yap Shum. versus Republica del Peri [decisión sobre Jurisdicción del 19 de junio de 2009. Caso CIADI ARB/07/6]; Renta 4 S.V.S.A y otros versus Rusiu, [decisión sobre objeciones preliminares de 20 de marzo de 2009. CCE No.024/2007], decisión de la mayoria del Tribunal con una opinión en disidencia; Roslmest UK Ltd sersus Rusia, [decisión sobre Jurisdicción de Octubre de 2007, Caso CCE].

75. Ver, en particular, Maffezini versus Reino de España. [decisiön sobre Jurisdicción de 25 de enero de 2000, Caso CIADI ARB/97/7)]; y, Siemens versus Arrentina, [decisión sobre Jurisdicción del 3 de agosto de 2004, Caso CIADI ARB/02/8]. 
hoc, lo que está generando importantes tensiones como consecuencia del surgimiento de múltiples disputas paralelas no acumulables con elementos fácticos y legales en común ${ }^{76}$. Argentina, por ejemplo, ha enfrentado más de casi 40 casos paralelos con estas características, todos ellos surgidos a partir de las medidas de emergencia adoptadas en el año 2001, con los costos e incertidumbres que ello implica.

Las nuevas generaciones de $\mathrm{TBIs}^{77}$ incluyen disposiciones sobre acumulación de procedimientos y presentan innovaciones y precisiones procesales que, aunque un tanto excesivas en su reglamentación, demuestran una creciente preocupación en delinear y precisar los pormenores operativos de los mecanismos de solución de controversias entre inversores y estados. El tiempo dirá si esta es la dirección correcta. Mientras tanto, la jurisprudencia arbitral iluminará u oscurecerá el camino. En todo caso, esperemos que prime la visión crítica por sobre la complaciente, y que sendero que se talle esté iluminado por la vocación de fomentar el estado de derecho y no por los intereses políticos o profesionales contingentes.

76. Ver "Acumulacioin de Arbitrajes bajo Tratades Bilaterules de Inversion", C. Ignacio Suarez Anzorena. Lima Arbitration I. 2006. p. 113.

77. Ver, por cjemplo, TBI Perí - Canada y TLC Peru - Estados Unidos. 